\title{
Medical Volume Segmentation using Bank of Gabor Filters
}

\author{
Adebayo Olowoyeye \\ Department of Computer \\ Science \\ Indiana University \\ Bloomington, IN 47405-7104 \\ aolowoye@indiana.edu
}

\author{
Mihran Tuceryan \\ Department of Computer \& \\ Information Science, IUPUI \\ 723 W. Michigan St \\ Indianapolis, IN 46202, USA \\ tuceryan@iupui.edu
}

\author{
Shiaofen Fang \\ Department of Computer \& \\ Information Science, IUPUI \\ 723 W. Michigan St \\ Indianapolis, IN 46202, USA \\ sfang@cs.iupui.edu
}

\begin{abstract}
In this paper, we will present an unsupervised approach for segmenting medical volume images based on texture properties. The texture properties of the volume data are defined based on spatial frequencies as implemented using a statistical method known as Gabor filters. Each Gabor filter in the bank is tuned to detect patterns of a specific frequency and orientation when convolved with a medical volume. The convolution is performed in the Fourier domain and the resulting response image is a feature which is added to our feature vector. The feature vector is thus passed into a classification/segmentation algorithm.
\end{abstract}

\section{Categories and Subject Descriptors}

I.4.6 [Segmentation]; I.4.7 [Feature Measurement]: Texture; I.4.9 [Applications]; J.3 [Life and Medical Sciences]: Health

\section{General Terms}

Algorithm, Design, and Experimentation

\section{Keywords}

Segmentation, Classification, Gabor Filters, K-Means, Clustering, Texture Analysis, Medical Volume Images

\section{INTRODUCTION}

Computer based medical diagnosis applications play a critical role in aiding health care specialists when trying to understand and diagnose anatomical structures. Medical volume segmentation is a vital part of any computer based medical diagnosis software and can fall into three categories: structural techniques, statistical techniques, and hybrid techniques. Structural techniques perform poorly when noise is present or the input data has low contrast. They are also very difficult to automate so manual intervention is typically needed. Statistical segmentation techniques involve

Permission to make digital or hard copies of all or part of this work for personal or classroom use is granted without fee provided that copies are not made or distributed for profit or commercial advantage and that copies bear this notice and the full citation on the first page. To copy otherwise, to republish, to post on servers or to redistribute to lists, requires prior specific permission and/or a fee.

SAC'09 March 8-12, 2009, Honolulu, Hawaii, U.S.A

Copyright 2009 ACM 978-1-60558-166-8/09/03 ...\$5.00. using algorithms that segment based on statistical analysis of image features. Segmentation is accomplished by creating features derived from the input volume. These features are then used to partition the image space according to a dissimilarity criterion. A feature space is the range of an $\mathrm{N}$-dimensional feature vectors computed from features at each voxel [3]. Examples of features are the voxel intensity, the gradient at the voxel, distance from the boundary, etc. Once the feature vector is built, one can then use a variety of clustering algorithms (k-means, fuzzy clustering) to group similar pixels together, resulting in the segmented image. In this paper, we are interested in medical volume images and their segmentation into meaningful regions based on their textural properties. Medical volume segmentation is considered a difficult problem due to the size of the input data, the subtleties that distinguish some tissues and the complexity of anatomical structures [8]. As a result the computational burden of implementing segmentation techniques is high. Another issue with many medical volume segmentation techniques is that they are supervised, meaning they rely heavily on human interaction. The ultimate goal is to have a robust, unsupervised, and efficient segmentation technique that can receive any medical volume data as input. Our research aims to attain this goal by using a Gabor filter bank to segment medical volumes using the volume's texture property alone.

\section{RELATED WORK}

Medical image segmentation is one of the most actively studied fields in the past few decades. The number of segmentation algorithms found in literature is very high. These segmentation algorithms are often specific to a particular problem, thus having little significance for other problems. Duncan and Ayache describe the progress of medical image analysis over two decades [5]. As the development of modern imaging modalities such as magnetic resonance imaging (MRI) and computer tomography (CT), physicians and technicians nowadays have to process the increasing number and size of medical images. Therefore, efficient and accurate computational segmentation algorithms become necessary to extract the desired information from these large data sets. Most medical segmentation algorithms are for two dimensional medical images (2D Slice), so they translate poorly to three dimensional medical images. The algorithms are also intensity based and thus do not rely significantly on spatial information. These algorithms do not work well when different regions have only subtle differences. In some cases, subtle patterns in the spatial arrangement of intensity 
values may be the only way to distinguish between the two regions. To address these issues, our algorithm segments medical volumes by characterizing texture properties using Gabor filters.

Gabor filters are a multi-channel filtering approach that is inspired by research in psychophysiology. These studies suggest that the brain performs a multi-channel, frequency and orientation analysis of the visual image formed on the retina. De Valois et al. recorded the response of the simple cells in the visual cortex of a macaque monkey to sinusoidal gratings of various frequencies and orientations. They discovered that these cells are tuned to narrow ranges of frequency and orientations [8]. This showed that groups of cells in the visual cortex are specialized to recognize patterns within a specific orientation and frequency range. These studies in psychophysiology have motivated the application of multichannel filtering approaches to texture analysis and have proven very effective. The 1-D Gabor Filter was first introduced by D. Gabor in 1946. The filter collection originally proposed by Gabor was extended to the two-dimensional case by Daugman [4]. Bovik et al. were influential in the early 1990's by demonstrating the reliability of texture segmentation schemes based on the multi-channel approach using 2D Gabor functions as localized spatial filters [1]. Bovik et al. is a basis over which more complex texture segmentation systems are built. Jain and Farrokhnia [7] implemented an unsupervised texture segmentation and classification technique for 2D images that use a bank of 2D Gabor filters. This showed that $2 \mathrm{D}$ images can be segmented and then classified using its texture properties alone. Gabor filters have been successfully applied to a wide range of other image processing applications such as image retrieval, document analysis, image coding, retina identification, character recognition, edge detection, medical image compression and image representation.

\section{OVERVIEW OF ALGORITHM}

Our algorithm uses a statistical approach to segmentation, where a bank of Gabor filter is used to characterize textural components of a medical volume. Figure 1 shows the segmentation process.

The input image is convolved with each filter in the Gabor filter bank. The resulting response image is added to the feature vector that is passed to a classifying algorithm. The result from the classification algorithm is the segmented image.

\subsection{Gabor Filters}

While the use of Gabor filters is widespread, the design of these filters is still an open issue. There are two main approaches to the selection of Gabor filters. One approach is to use a large bank of filters and the second is to design a small set of customized filters. Our program uses the former approach because we want a robust system that can analyze most volume types. The formula for a complex Gabor function in space domain is

$$
g(x, y, z)=s(x, y, z) w_{r}(x, y, z)
$$

where $s(x, y, z)$ is a complex sinusoidal, known as the carrier, and $w_{r}(x, y, z)$ is a 3-D Gaussian-shaped function, known as the envelop. The complex sinusoidal is defined as follows,

$$
s(x, y, z)=\exp \left(j\left(2 \pi\left(u_{0} x+v_{0} y+w_{0} z\right)+P\right)\right)
$$

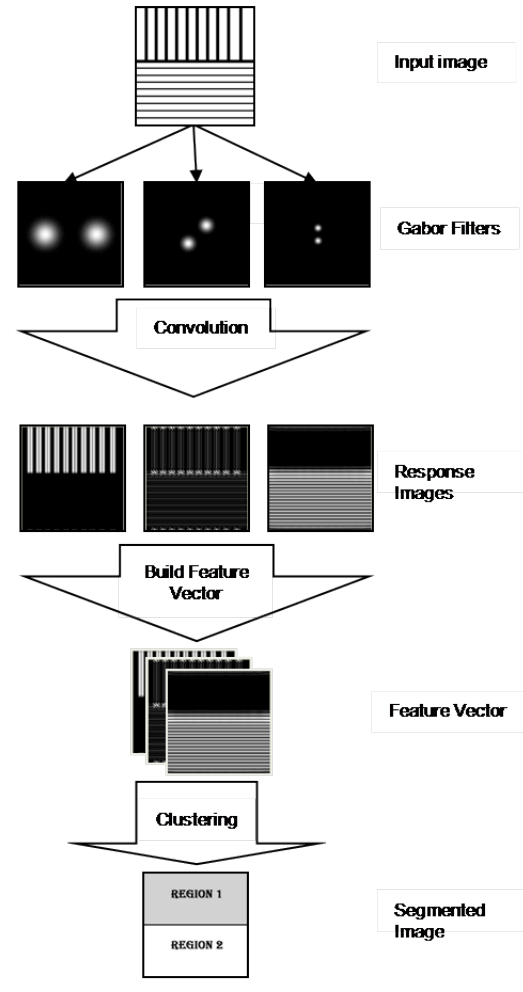

Figure 1: An overview of the segmentation algorithm.

where $\left(u_{0}, v_{0}, w_{0}\right)$ and $P$ define the spatial frequency and the phase of the sinusoidal respectively [9]. The sinusoid has a real component and an imaginary component.

$$
\begin{aligned}
& \operatorname{Re}(s(x, y, z))=\cos \left(2 \pi\left(u_{0} x+v_{0} y+w_{0} z\right)+P\right) \\
& \operatorname{Im}(s(x, y, z))=\sin \left(2 \pi\left(u_{0} x+v_{0} y+w_{0} z\right)+P\right)
\end{aligned}
$$

The parameters $u_{0}, v_{0}$ and $w_{0}$ define the spatial frequency of the sinusoidal in Cartesian coordinates. The Gaussian envelope is

$$
w_{r}(x, y, z)=K \exp \left[-\pi\left(a^{2}\left(x-x_{0}\right)_{r}^{2}+b^{2}\left(y-y_{0}\right)_{r}^{2}+c^{2}\left(z-z_{0}\right)_{r}^{2}\right.\right.
$$

where $\left(x_{0}, y_{0}, z_{0}\right)$ is the peak of the function, $a, b$ and $c$ are scaling parameters of the Gaussian, and the $r$ subscript stands for a rotation operation. This allows us to have Gabor filters with different orientations. The complexity of convolution depends directly on the size of the convolution mask. The complexity of calculation the filter response for one voxel is $O\left(M^{3}\right)$ where $M$ is the width, height and depth of the convolution window. The complexity of calculating the whole image in spatial domain is $O\left(M^{3} N^{3}\right)$, where $N$ denotes the width, height and depth of the volume.

The convolution is computed much more efficiently by using the convolution theorem and working in the frequency domain. The complexity of 3-D FFT and IFFT is $O\left(N^{3} \log N\right)$. The total complexity for calculating the filter response in frequency domain is $2 * O\left(N^{3} \log N\right)+N$. Convolution of our input volume and Gabor filters is more computationally expensive in the spatial domain, so we convolve them in the frequency domain. The 3-D Fourier transform of the Gabor 
filter is

$$
\begin{aligned}
g(u, v, w)= & \frac{K}{a b c} \exp \left[j\left(-2 \pi\left(x_{0}\left(u-u_{0}\right)+y_{0}\left(v-v_{0}\right)+z_{0}\left(w-w_{0}\right)\right)\right)\right] \\
& \star \exp \left[-\pi\left(\left(\frac{\left(u-u_{0}\right) r}{a^{2}}+\frac{\left(v-v_{0}\right) r}{b^{2}}+\frac{\left(w-w_{0}\right) r}{c^{2}}\right.\right.\right.
\end{aligned}
$$

\subsection{Gabor Filter Bank}

A single Gabor filter will detect a pattern of a certain frequency and orientation, but to characterize complex texture patterns one must systematically capture all orientation and frequency components. We use a bank of Gabor filters where each filter in the bank is fine tuned to detect patterns of different frequencies and orientations. This gives us multiple Gabor filters that collectively represent all possible texture patterns. This is important because relationships between filter responses provide the basis for classification or segmentation.

The design of a Gabor filter bank is dependent on the optimal values for a filter's frequency, bandwidth and the number of orientations. The number of different frequencies in the bank of Gabor filters is determined by the size of the input volume. The highest radial frequency that guarantees the pass band of the filter falls within the image is $\left(\frac{N}{4}\right) \sqrt{2}$ cycles/volume-width, where the volume has a width of $\mathrm{N}$ voxels [2]. Valid radial frequencies are:

$$
1 \sqrt{2}, 2 \sqrt{2}, 4 \sqrt{2}, \ldots, \text { and }\left(\frac{N}{4}\right) \sqrt{2}-\text { cycles/volume width }
$$

The frequency bandwidth, in octaves, from frequency $f_{1}$ to frequency $f_{2}$ is given by $\log _{2}\left(\frac{f_{2}}{f_{1}}\right)$; so radial frequencies are 1 octave apart. This is based on studies in psychophysiology that have shown that frequency bandwidth of simple cells in the visual cortex is about 1 octave [2]. Filters with very low radial frequencies, $1 \sqrt{2}$ and $2 \sqrt{2}$ cycles/volume width, can often be left out because they capture spatial variations that are too large to correspond to texture [2]. There is no hard and fast rule to the number of orientations, but the goal is to cover all the frequency space with little overlapping between Gabor filters. The figure below shows an ideal design for two adjacent filters with different orientations, but with the same frequency [9]. $f_{0}$ is the filter frequency and $\alpha$ is the orientation.

The figures below show two filter banks in the frequency space. In Figure 2, there are large gaps between filters in different orientation, and as a result there can be features at specific angles that cannot be detected by the filter bank. The filter in Figure 3 is more robust because there are fewer gaps between filters so more features are detectable [9].

\section{IMPLEMENTATION DETAILS}

$\mathrm{Oh}$ and Choe performed extensive research on the nature of textures. They examined texture segmentation in $2 \mathrm{D}$ versus $3 \mathrm{D}$. They found that textures naturally define distinct physical surfaces, and thus the ability to segment texture in 2D may have grown out of the ability to distinguish surfaces in $3 \mathrm{D}$. To test this insight they compared texture segmentation performance of two neural networks trained on textures arranged in both $2 \mathrm{D}$ and $3 \mathrm{D}$. Their results revealed that texture segmentation is easier to learn in $3 \mathrm{D}$ than in $2 \mathrm{D}$, and furthermore the network trained in 3D easily resolved the $2 \mathrm{D}$ problem as well, but not the other way around [10]. Our program is built on a Linux platform and is developed in $\mathrm{C}++$. We use the Visualization Toolkit (VTK) for volume

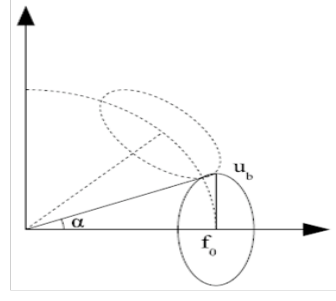

Figure 2: Two Gabor filters with different orientations in the frequency space.

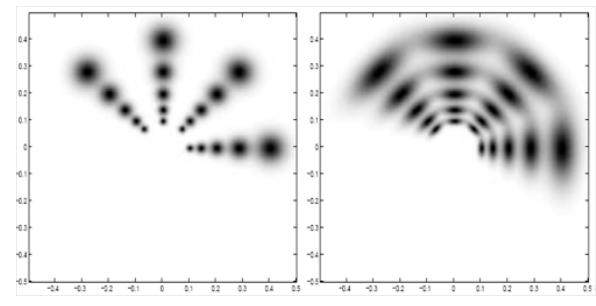

Figure 3: Gabor filter bank in two dimensions.

\begin{tabular}{|r|r|r|r|r|r|}
\hline $\begin{array}{r}\text { volume } \\
\text { dims }\end{array}$ & $\begin{array}{r}\text { \# of } \\
\text { voxels }\end{array}$ & byte & $\begin{array}{r}\text { size } \\
(\mathrm{MB})\end{array}$ & $\begin{array}{r}\text { \# of } \\
\text { filter }\end{array}$ & $\begin{array}{r}\text { memory } \\
(\mathrm{MB})\end{array}$ \\
\hline 64 & 262144 & 4 & 1 & 39 & 40 \\
128 & 2097152 & 4 & 8 & 52 & 424 \\
256 & 16777216 & 4 & 64 & 65 & 4224 \\
512 & 134217728 & 4 & 512 & 78 & 40448 \\
1024 & 1073741824 & 4 & 4096 & 91 & 376832 \\
\hline
\end{tabular}

Table 1: Resource requirements based on input size.

manipulation and Qt, Trolltech's cross-platform application development for the user-interface.

\subsection{Input}

Input files that are currently analyzed by the program are either an MRI or CT Scan volumes. However, the program is very robust and can segment any type of volume. The program can load file formats such as AVI, Bitmap, DICOM, JPEG, PNG, TIFF and even RAW files. The input volume is stored in a VTK data structure. The size of the input volume varies, so to cut down on the complexity we pad input volumes to have sizes as powers of two in all axes. The volumes can be as small as $64^{3}$ and as large as $1024^{3}$. Our experiments use MRI volumes of the brain as input data with volume sizes ranging between $128^{3}$ and $256^{3}$. We are restricted to this range because computing resources grow exponentially as we increase the input size. When we increase input size we need a larger Gabor filter bank and a larger feature vectors.

\subsection{Design of Gabor Filter Bank}

The bank of Gabor filters is pre-built and stored as a binary file. Gabor filters need to be the same size as the input image so we have created sets of filter banks with different sizes (all filter sizes are a power of two). We are using four different orientations and as discussed in section two, the number of possible frequencies is determined by the size of 
the input image. If $\left(\frac{N}{4}\right) \sqrt{2}$ is the highest frequency, then the number of frequency available at a volume size is $\log _{2}\left(\frac{N}{4}\right)$. The radial frequencies $1 \sqrt{2}$ and $2 \sqrt{2}$ (cycles/volume width) capture spatial variations that are too large so they are not added to the filter bank, so the equation for the number of frequencies available for a specific volume size is:

$$
F_{n}=\log _{2}\left(\frac{N}{4}\right)-2
$$

The total number of filters in our bank is:

$$
N_{f}=\left(F_{n} \times a_{x} \times a_{y} \times a_{z}\right)-3 F_{n}
$$

where $a_{x}, a_{y}$, and $a_{z}$ represents the number of orientations at each axis $\left(a_{x}=a_{y}, a_{z}=1\right)$. The second part of equation 8 removes duplicate filters from the bank. For a volume of size $64^{3}$ we need a Gabor filter bank with 39 filters of the same size. A volume size of $1024^{3}$ needs a Gabor filter bank with 91 Gabor filters.

\subsection{Feature Vector}

To create the feature vector for a volume it must be transformed to the frequency domain by using Fast Fourier Transform (FFT). We use the FFTW $\mathrm{C}++$ library to calculate the transformation. Once in frequency domain we do a piecewise multiplication of our volume and a filter. The response from the convolution is transformed back to spatial domain via IFFT (inverse FFT) and then added as a feature in the feature vector. Each feature in the vector is essentially a dimension in our feature space. For $\mathrm{N}$ filters in the filter bank, each voxel in the image volume will be represented by an N-dimensional feature vector, each component of which is the response to the corresponding Gabor filter.

\subsection{Classification and Segmentation}

Once the feature vector is computed, it is passed to a clustering algorithm. We use the k-means clustering or fuzzy clustering algorithm to group similar voxels. We also experimented with fuzzy clustering and found that it gives better results. The results from clustering can then be displayed for further interactions by the user. The user can interact with the segmented volume by viewing each cluster independently or by viewing all segmented clusters at once.

\section{RESULTS}

We have tested our algorithm on multiple MRI volumes of the human head, CT Scans of human head, MRI volumes of a frog and an MRI volume of a human leg. To demonstrate the performance of our segmentation algorithm, we will us a $128^{3}$ MRI volume of a humans brain. Since the volume has a size of $128^{3}$, we need 52 Gabor filter in our bank to characterize all possible frequency and orientation components. Each filter in the bank is tuned to an orientation with an $\mathrm{x}, \mathrm{y}$ and $\mathrm{z}$ component and to one of four highest radial frequencies $(4 \sqrt{2}, 8 \sqrt{2}, 16 \sqrt{2}, 32 \sqrt{2})$. The algorithm is not completely unsupervised because the user must input the number of segments within the medical volume. When a number of segments is selected that is less than an ideal number, regions of similar texture are grouped together. When a segment number is selected greater than the ideal number, regions with the same textures are further segmented. This demonstrates that the algorithm is using texture properties to successfully segment regions with similar texture properties. We shared our results with two radiologists and they concluded that our algorithm does successfully segment the volume into anatomically meaningful regions.

\section{CONCLUSION}

Our research has successfully demonstrated that textural properties in medical volume images can be used to segment them into meaningful regions. Our algorithm is strong when segmenting images with high contrast between region, but its strength is in segmenting regions with subtle differences in intensity values and texture. The application was tested by two radiologists to verify that segmented regions are anatomically correct. We are currently working on a parallel programming implementation of our algorithm. This will decrease run-time significantly and will allow us to use more Gabor filters. Future work will also include experiments on finding the optimal number of Gabor filters for each image size. This will lead to a more accurate characterization of texture properties.

\section{REFERENCES}

[1] M. C. A.C. Bovik and W. Geisler. Multichannel texture analysis using localized spatial filters. IEEE Transactions on Pattern Analysis and Machine Intelligence, 12:55-73, 1990.

[2] J. Bigun. Speed, frequency, and orientation tuned 3-d gabor filter banks and their design. In IAPR Int'l Conf. on Pattern Recognition, volume c, pages 184-187, 1994.

[3] A. M. M. F. D. M. D. Chute and J. Tomaszewski. A novel stochastic combination of $3 \mathrm{~d}$ texture features for automated segmentation of prostatic adenocarcinoma from high resolution mri. Medical Imaging Computing and Computer-Assisted Intervention, 2:581-591, 2003.

[4] J. G. Daugman. Uncertainty relation for resolution in space, spatial frequency and orientation optimized by two-dimensional visual cortical filters. Journal of Optical Society of America, 2:1160-1169, 1985.

[5] J. Duncan and N. Ayache. Medical image analysis: Progress over two decades and the challenges ahead. In IEEE Transactions on Pattern Analysis and Machine Intelligence, pages 85-106, 2000.

[6] M. Feng and T. Reed. Dense motion field estimation by 3 -d gabor representation. In ICIP International Conference on Image Processing, volume 4, pages 2555-2558, October 2004.

[7] A. Jain and F. Farrokhnia. Unsupervised texture segmentation using gabor filters.

[8] S. Lakare. 3D Segmentation Techniques for Medical Volumes. December 2000.

[9] A. M. M. Fernandez and M. Tejera. Texture segmentation of a $3 \mathrm{~d}$ seismic section with wavelet transform and gabor filters. In IAPR Intl Conf. on Pattern Recognition, volume 3, pages 3358-3361, 2000.

[10] S. Oh and C. Yoonsuck. Texture segmentation in $2 \mathrm{~d}$ vs. 3d: Did 3d developmentally precede $2 \mathrm{~d}$ ?

[11] D. M. Z. Qian and L. Axel. Extraction and tracking of mri tagging sheets using a $3 \mathrm{~d}$ gabor filter bank. In Proceedings of Intl Conf. of the Engineering in Medicine and Biology Society, 2006. 


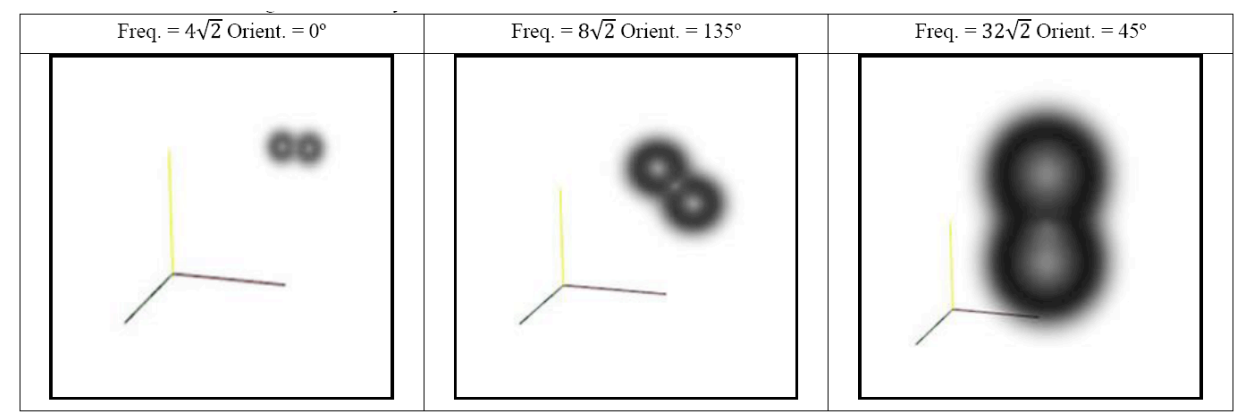

Figure 4: Sample Gabor filters: Gabor filters are created in frequency domain and these values are stored in a binary file. Filters with low frequency values capture textures with higher repetition and filters with high frequency values capture textures with lower repetition.

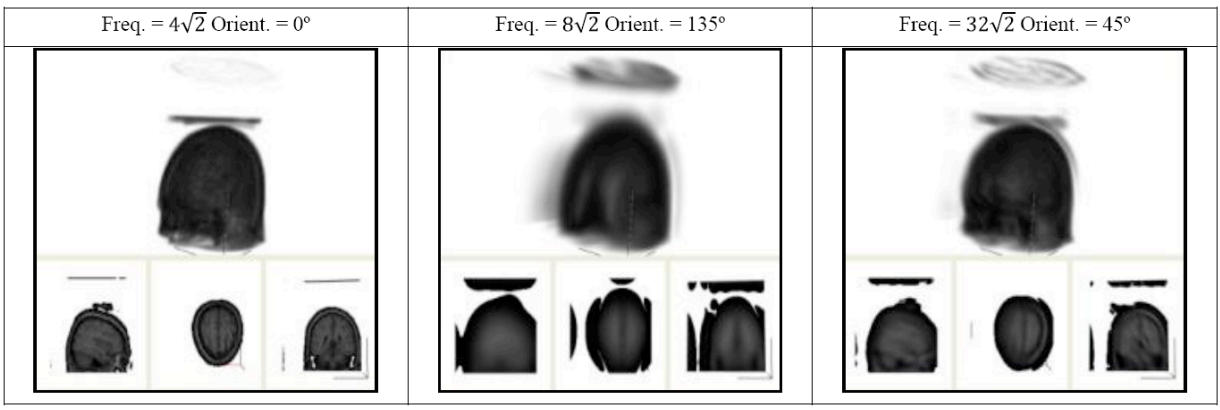

Figure 5: Features in Spatial Domain: Convolution between the input volume and Gabor filters are performed in the frequency domain. The resulting response images are converted back to the spatial domain to produce the images below. These images are used to construct the feature vector, where each response is a feature in the vector.

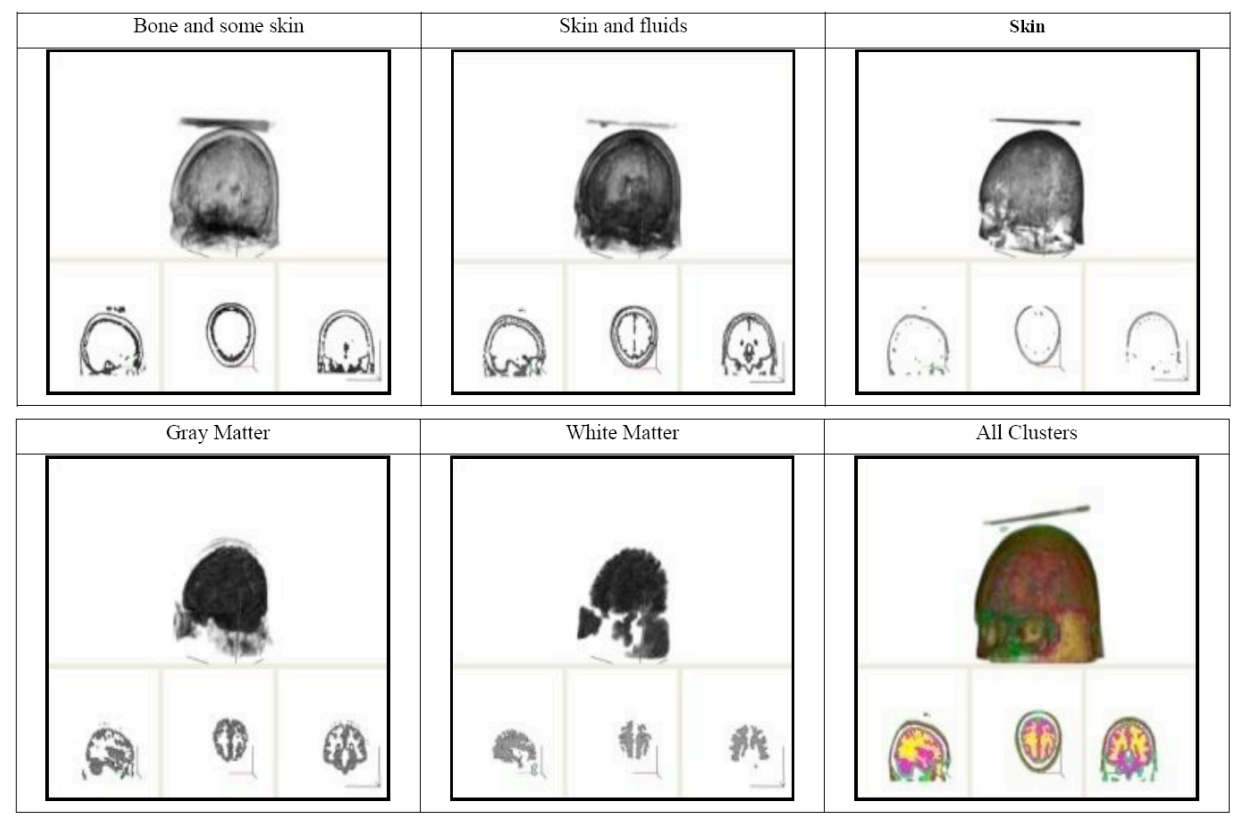

Figure 6: Clustering/Segmentation Results: For the following results, we set the segment number to five and, as verified by the radiologist, the program segments the MRI brain volume into the following regions: bone, fluids, skin, white matter and gray matter. There are some crossovers between the tissues, but this is improved with strict ending conditions for the clustering algorithm. The drawback to this is a longer runtime for the program. 